\title{
The use of bisphosphonates in women prior to or during pregnancy and lactation
}

\author{
Stathopoulos P. Ioannis, Liakou G. Chrysoula, Katsalira Aikaterini, Trovas George, \\ Lyritis P. George, Papaioannou A. Nikolaos, Tournis Symeon
}

Laboratory for the Research of Musculoskeletal System “Th. Garofalidis”, KAT Hospital, Athens, Greece

\begin{abstract}
OBJECTIVE: The unique pharmacokinetics of bisphosphonates (BPs) in conjunction with their use by an increasing number of women at reproductive age has raised serious concerns about their safety during pregnancy and lactation. Bisphosphonates cross the placenta. Animal studies have shown adverse effects on both the fetus and the mother, mostly at doses much higher than those commonly used in humans. Protracted parturition, maternal mortality, embryolethality, severe general underdevelopment and marked skeletal retardation of the fetuses (increased amount of diaphyseal bone trabeculae, decreased diaphyseal length), small fetal weight and abnormal tooth growth have been observed. DESIGN: We conducted a thorough research of the literature in order to identify human studies concerning this issue. RESULTS: We identified a total of 78 cases involving fetuses whose mothers had been exposed to BPs before conception or during pregnancy, along with 7 cases of BPs exposure prior to or during lactation. The vast majority of mothers and infants did not demonstrate serious adverse effects. However, there were cases of shortened gestational age, low neonatal birth weight and transient hypocalcaemia of the newborns, while the very few reported cases of spontaneous abortions and congenital anomalies probably resulted from maternal underlying diseases and concomitant medication. CONCLUSION: The administration of bisphosphonates in pregnancy should be assessed in view of their potential hazardous effects on both mother and fetus. In cases of absolute or relative indications of BPs prior to pregnancy, close observation of the mother and the infant, especially during the first two weeks of life, is imperative for the successful outcome of pregnancy.
\end{abstract}

Key words: Bisphosphonates, Lactation, Pregnancy

Address for correspondence:

Symeon Tournis M.D., PhD, Laboratory for the Research of Musculoskeletal System "Th. Garofalidis", KAT Hospital,

10 Athinas Str., 14561, Kifissia, Athens, Greece,

Tel.: +302108018123, Fax: +302108018122,

E-mail: stournis@med.uoa.gr

Received 28-07-11, Revised 27-08-11, Accepted 10-09-11

\section{INTRODUCTION}

Bisphosphonates (BPs) are the most common drugs used for the treatment of osteoporosis, with more than 29 million prescriptions issued during 2006 in the USA. ${ }^{1}$ BPs are also administered, with excellent results, in patients with hypercalcaemia, metastatic 
bone disease (e.g. due to breast, lung, prostate cancer and multiple myeloma) and Paget disease, while BP treatment in other conditions characterized by high bone turnover such as osteogenesis imperfecta and fibrous dysplasia has been shown to reduce skeletal morbidity. ${ }^{2}$ Nevertheless, although the vast majority of patients treated with BPs are postmenopausal women, the plethora of indications for BPs use has resulted in an increasing number of children, men and premenopausal women being treated with them.

Animal studies have demonstrated that BPs given to pregnant rats cross the placenta and result in reduced bone growth and fetal weight. ${ }^{3}$ Furthermore, high-dose (up to 10 to $15 \mathrm{mg} / \mathrm{kg} /$ day), long-term BP administration to pregnant rats and rabbits shows significant maternal and offspring toxicity, mainly due to protracted parturition and hypocalcaemia in dams, this susceptible to prevention by concomitant intravenous calcium supplementation. ${ }^{4}$ Moreover, $\mathrm{BP}$ administration at doses 10 times higher than human therapeutic doses resulted in embryolethality or severe underdevelopment and marked skeletal retardation. ${ }^{5}$ These facts along with BPs long-term skeletal retention have given rise to serious considerations about the safety of BPs use in women of reproductive age due to the possible adverse effects on an eventual pregnancy (synchronous with or subsequent to treatment).

With the aim of recapitulating the knowledge acquired so far regarding the administration of BPs prior to or during pregnancy and lactation, we conducted a thorough research of the literature in order to identify reports concerning this issue.

\section{METHODS}

We performed our research through PubMed using the words "bisphosphonates" and "pregnancy" or "bisphosphonates" and "lactation" as key words. The initial number of articles that matched our criteria was 120. After the exclusion of those concerning animal or laboratory studies, reviews not adding new cases, cases with no exposure of the fetus or the infant to BPs and those where the fetus or the infant was exposed to BPs but not providing information about the outcome, the number of articles was reduced to 18 .

We also searched the published trials of BPs administered for the prevention and treatment of glucocorticoid induced osteoporosis, Paget's disease and premenopausal osteoporosis. In these publications there was no report of BPs exposure during pregnancy and/or lactation. We did not include oncology patients in trials investigating the effect of BPs, such as zoledronate or pamidronate, on disease-associated events. However, we included single case reports of women with malignancy-associated hypercalcaemia due to breast cancer diagnosed during pregnancy and treated with BPs.

\section{RESULTS}

Seventy-eight cases of fetuses whose mothers had been exposed to BPs before conception or during pregnancy were identified, along with 7 cases of BPs exposure prior to or during lactation (Table 1). Alendronate p.o. and i.v. pamidronate were the commonest BPs used. Mean maternal age at conception was 32.2 years. Duration of BPs use prior to pregnancy ranged from 5 years to only 6 months. However, in 59 cases duration of prior BP exposure was not reported. In most cases treatment was stopped 3 months prior to or at conception, although there were at least 33 cases that received BPs during the first 3 months of pregnancy. Only a small number of women received BPs after the first trimester, mainly due to malignancy associated hypercalcaemia. Indications for BP administration included glucocorticoid-induced osteoporosis, pregnancy-associated osteoporosis, autoimmune diseases, osteogenesis imperfecta and polyostotic fibrous dysplasia and malignancy-associated hypercalcaemia. Mean gestational age was 36.5 weeks and mean birth weight was $\sim 2.7 \mathrm{~kg}$. Sixty-nine pregnancies resulted in live births, while 9 abortions (8 spontaneous, 1 voluntary) were noted; all of these abortions were attributed to maternal concomitant diseases and medication.

\section{a. BPs prior to or during pregnancy}

\section{- Large case-series}

The largest case-series study to date of pregnant women exposed to BP was reported by Ornoy et al. They studied the outcome of pregnancy in 24 women who were exposed to alendronate before conception or during the first months of pregnancy. These women were suffering from osteoporosis secondary 


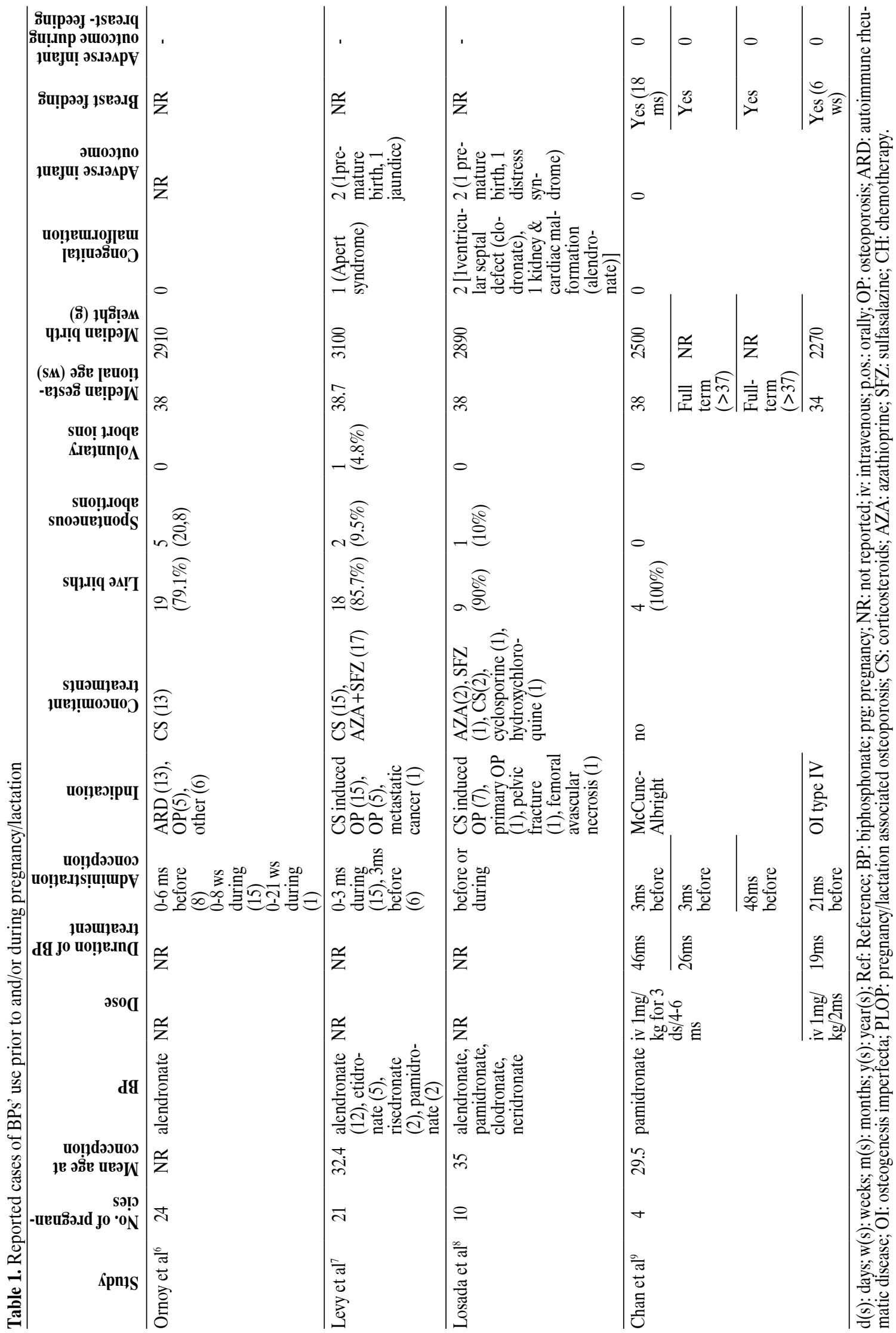




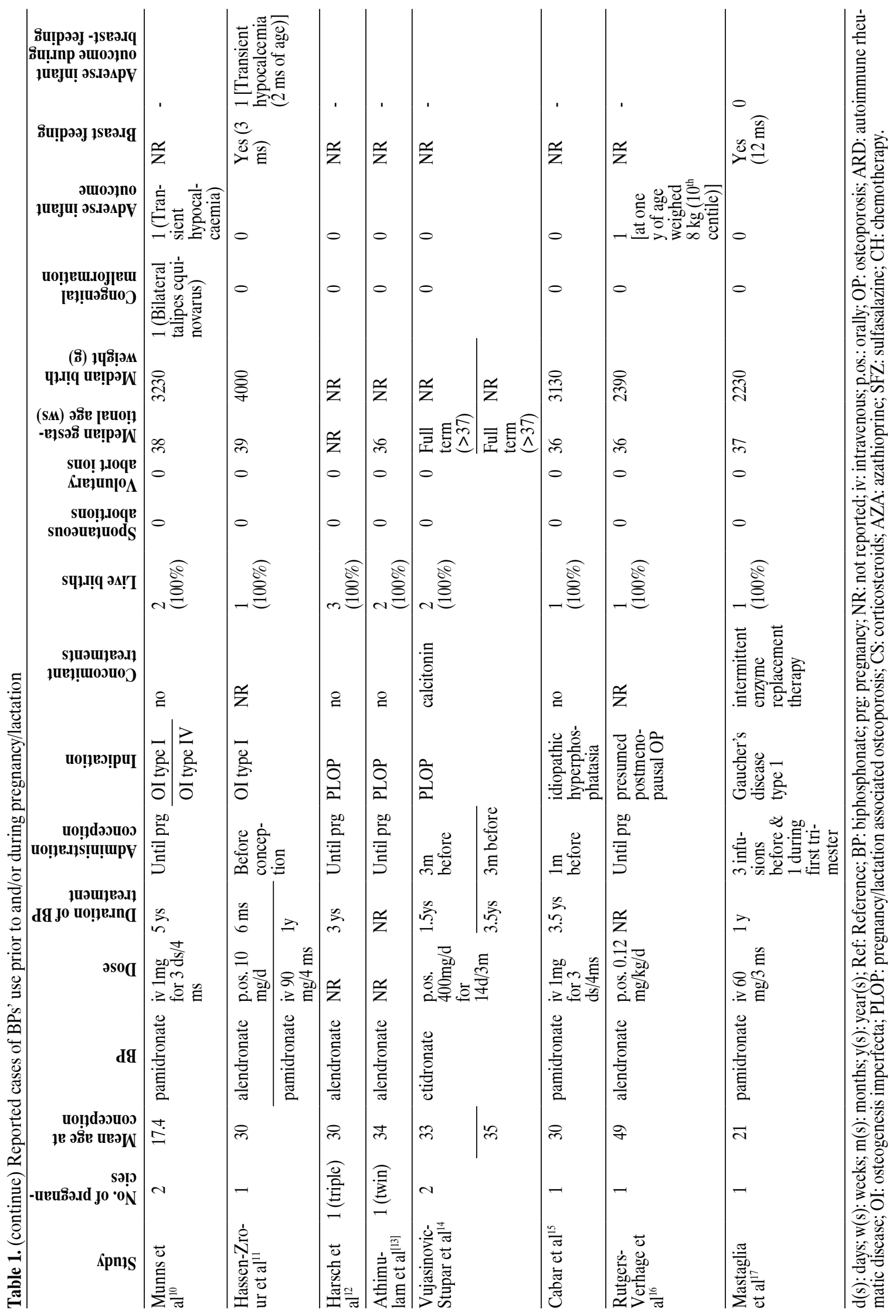




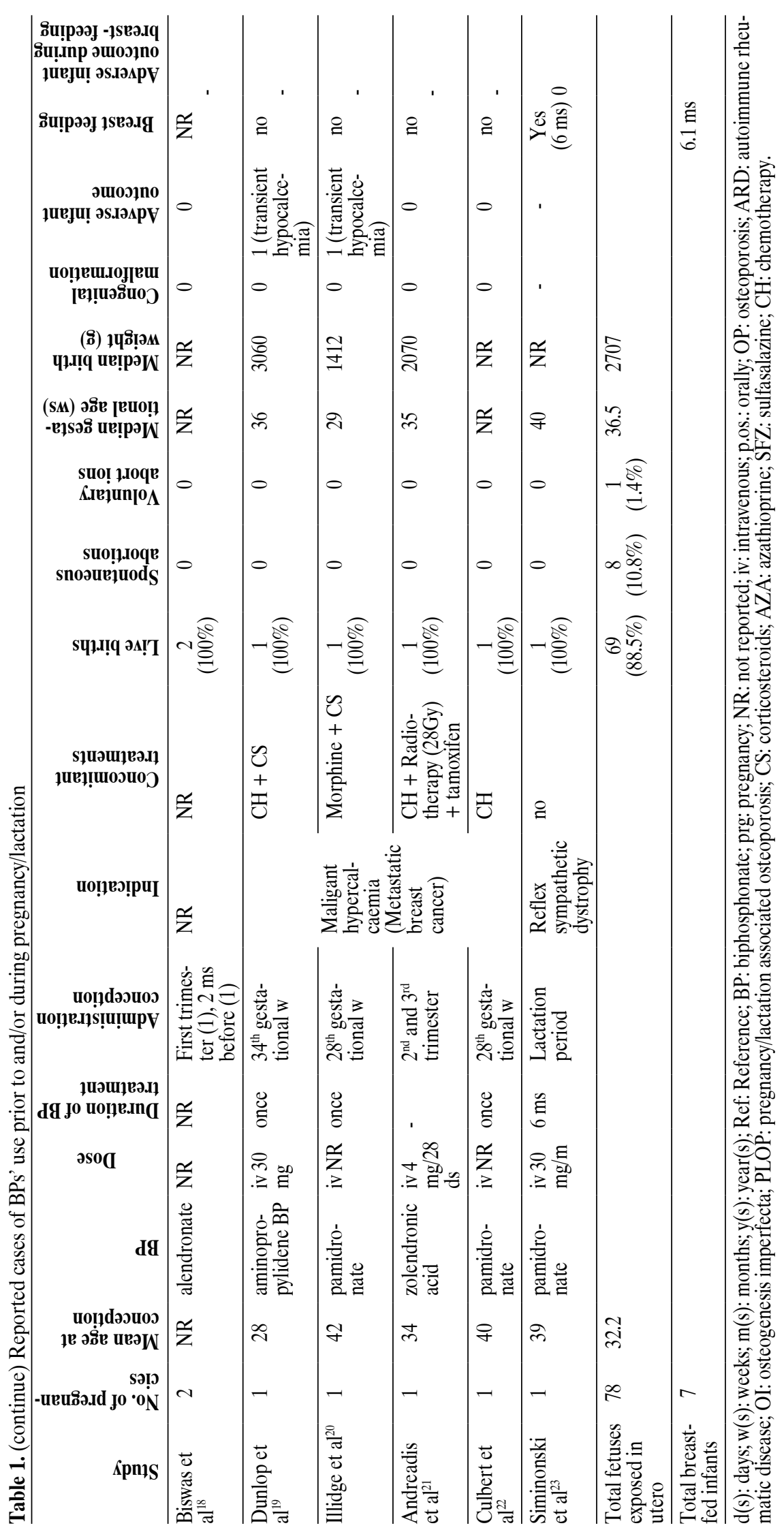

to major chronic disease and/or the medication against it. The diagnoses of the patients were as follows: systemic lupus erythymatosus (SLE) $(\mathrm{n}=5)$, familial osteoporosis $(n=4)$, Takayasu arteritis $(\mathrm{n}=2)$, AdamantiadisBehcet's disease $(n=2)$, hypothyroidism $(n=2)$, rheumatoid arthritis $(\mathrm{n}=1)$, psoriatic arthritis $(n=1)$, Crohn's disease $(\mathrm{n}=1)$, asthma $(\mathrm{n}=1)$, berylliosis $(\mathrm{n}=1)$, autoimmune hepatitis $(n=1)$, early menopause $(n=1)$, Laron syndrome $(n=1)$, leprosy with perinatal listeria infection $(n=1)$. The investigators found significantly lower median gestational age and birth weight compared to the control group, while the rate of spontaneous abortions was higher. However, these findings may be related to the maternal diseases responsible for osteoporosis or to the additional drugs taken, especially glucocorticoids. Among the offspring, no major congenital anomalies were observed. ${ }^{6}$

Levy et al performed a multicenter prospective cohort study including 21 women exposed to BPs during pregnancy or even 12 months before conception, due to primary or secondary osteoporosis, and 21 matched controls. Fifteen patients in the BPs group had first-trimester exposure and 6 patients discontinued the bisphosphonates within 3 months prior to conception, but further information about time of exposure was not reported. BPs used were as follows: alendronate $(\mathrm{n}=12)$, etidronate $(n=5)$, risedronate $(n=2)$ and pamidronate $(\mathrm{n}=2)$. The indica- 
tions were primary osteoporosis $(\mathrm{n}=5)$, osteoporosis associated with cancer $(n=1)$ and osteoporosis secondary to corticosteroid use, such as inflammatory bowel disease and SLE $(n=15)$. Seventeen patients were on other medications during pregnancy because of these medical conditions, including azathioprine and sulfasalazine. The study's primary endpoint was neonatal outcome including major birth defects, while the secondary was the outcome of pregnancy (live births, spontaneous abortions, therapeutic abortions). There were no significant differences between the two groups in any outcome. One child, whose mother discontinued etidronate 3 months before conception, was born with Apert syndrome, an autosomal dominant acrocephalosyndactyly. However, it was not related to the medication as a linked gene mutation was found. ${ }^{7}$

Moreover, a recent review, that includes the aforementioned cohorts, adds data of 10 more women (mean age at conception $35 \mathrm{yrs} /$ range 30-43 yrs) exposed to BPs (alendronate, pamidronate, clodronate, neridronate) before or during pregnancy. The indications for BP included corticosteroid induced osteoporosis in 7 cases, primary osteoporosis in 1, pelvic fracture in 1 and femoral avascular necrosis in 1. Other concomitant treatments were: azathioprine in 2 cases, sulfasalazine in 1 , corticosteroids in 2 , cyclosporine in 1 and hydroxychloroquine in 1 . There was 1 spontaneous abortion and 9 newborns of mean gestational age 38 weeks and mean birth weight $2890 \mathrm{~g}$. Two congenital malformations, 1 ventricular septal defect (exposed to clodronic acid) and 1 kidney and cardiac malformation (exposed to alendronate), 1 premature birth and 1 distress syndrome were reported. ${ }^{8}$

\section{- Cases of women treated with BPs due to osteogenesis imperfecta (OI) or polyostotic fibrous dysplasia (PFD)}

Chan et al presented 4 infant outcomes of pregnancies of 3 women who were treated with intravenous pamidronate for 19-46 months before conception, 2 due to PFD, at a dose of $3 \mathrm{mg} / \mathrm{kg}$ every 4 months and 1 due to OI, at a dose of $1 \mathrm{mg} / \mathrm{kg}$ every 2 months. All of them had stopped medication for at least 3 months before conception. There were no pregnancy-related complications nor biochemical or skeletal abnormalities among the offspring. ${ }^{9}$
Another report of 2 cases dealt with the pregnancies of 2 women with OI, types I and IV, who received intravenous pamidronate for 5 years before conception. The first conceived at the age of 17 years and then discontinued pamidronate, which she had been receiving at a dose of $1 \mathrm{mg} / \mathrm{kg}$ on each of 3 consecutive days every 4 months since the age of 12 . Throughout pregnancy she received daily $1000 \mathrm{mg}$ calcium and 400 IU vitamin D. The woman delivered a boy of $3.6 \mathrm{~kg}$ at 37 weeks of gestation. The boy had hypocalcaemia at 24 hours of age, but phosphorus and $\mathrm{PTH}$ concentrations were within normal range. On day 11 his calcium was normal. The boy inherited his mother's COL1A1 mutation. The second woman, who had been receiving pamidronate from 12.5 to 17.8 years of age, conceived at the age of 17.8 years and delivered a girl of $2.86 \mathrm{~kg}$ at 38 weeks, who also inherited her mother's disease and presented bilateral talipes equinovarus. She had no signs or symptoms suggestive of hypocalcaemia. Both babies presented normal growth and no fracture throughout the next few months after birth. ${ }^{10}$

Recently, Hassen-Zrour et al reported the case of a 30-year-old woman with a 1-year history of diabetes mellitus and OI type I, who received bisphosphonates (10 mg alendronate qd for 6 months followed by $90 \mathrm{mg}$ intravenous pamidronate every 4 months for 1 year) before conception. At 39 weeks of gestation she gave birth to a healthy female infant who weighed $4 \mathrm{~kg}$ and did not present any malformation or perinatal irritability due to hypocalcaemia. The baby was breastfed until she was 3 months old. The mother remained free of pain and fracture at 6 months postpartum. She did not exhibit bone loss during pregnancy and breast feeding. ${ }^{11}$

\section{- Cases of women treated with BPS due to pregnancy-associated osteoporosis (PO) and various cases}

A 30-year-old patient, suffering from osteoporosis since her first pregnancy and treated with alendronate, had a triple pregnancy 3 years later and stopped medication. The outcome of pregnancy was positive, though no further details were given about neonatal health. ${ }^{12}$ Following that report, Athimulam et al ${ }^{13}$ and Vujasinovic-Stupar et al ${ }^{14}$ presented the outcome of 2 more cases of BPs-exposed women due to PO. In the first case, ${ }^{13}$ a 34 -year-old woman treated with 
alendronate after her second delivery, discontinued BPs at the beginning of her third pregnancy. She had an uneventful pregnancy and delivered 2 healthy infants (the authors did not give information about duration of treatment, dose of alendronate and further follow-up of the newborns). Additionally, VujasinovicStupar et $\mathrm{l}^{14}$ reported the case of a woman treated with intermittent etidronate therapy ( $400 \mathrm{mg}$ qd for 14 days every 3 months) due to PO beginning at the age of 30 years after her first pregnancy. At the age of 33 years and after being treated with etidronate for 1.5 years, she got pregnant for the second time. Her last cycle of etidronate was 3 months before conception, pregnancy and labor were uncomplicated and the baby (a full-term boy) was healthy at birth with no neonatal complications, no obvious skeletal deformities or hypocalcaemia and normal DXA values at follow-up. After the second delivery, the woman continued etidronate therapy for another 2 years and became pregnant again. Her last cycle of etidronate was 3 months before conception. Pregnancy and labor were uneventful. The baby (a full-term girl) was healthy at birth and follow-up.

In another report, a 30-year-old woman, who had been receiving intermittently intravenous pamidronate for 3.5 years $(1 \mathrm{mg} / \mathrm{kg}$ for 3 consecutive days every 4 months) for the treatment of idiopathic hyperphosphatasia, got pregnant 1 month after the last administration. She gave birth to a healthy offspring at 36 weeks of gestation who did not present any clinical or biochemical abnormalities. ${ }^{15}$

An additional case report described a 49-year-old woman, amenorrheic for 2 years, who was receiving oral alendronate at a dose of $10 \mathrm{mg} /$ day for postmenopausal osteoporosis. She was unaware of the pregnancy until delivery at 36 weeks of gestation. She delivered a girl, weighing $2390 \mathrm{~g}\left(50^{\text {th }}\right.$ centile $)$, without obvious birth defects or laboratory abnormalities. At 1 year of age the infant's development was normal. ${ }^{16}$ Recently, the case of a young woman diagnosed with type 1 Gaucher's disease on intermittent enzyme replacement and multiple vertebral fractures treated with intravenous pamidronate $60 \mathrm{mg}$ every 3 months since the age of 20 was reported. While under treatment she became pregnant (total dose $240 \mathrm{mg}$ - last during the first trimester). She delivered at the $37^{\text {th }}$ week a healthy girl weighing 2230 gr by caesarean section due to preeclampsia. Finally, an observational cohort study, conducted in the UK using the technique of prescription event monitoring and aiming to monitor the safety of alendronate, included the reports of 2 pregnancies. One patient took alendronate in the first trimester, while the other had stopped taking alendronate 2 months prior to conception. There were no problems reported during the pregnancies and both infants were healthy. ${ }^{18}$

\section{- Cases of women treated with BPs due to malignancies}

BPs are also commonly prescribed in the treatment of hypercalcaemia of malignancy. So far, 4 reports of pregnant women and furnishing information about the pregnancy outcome have been published. The first case concerned a 24-year-old woman who received $30 \mathrm{mg}$ pamidronate at 34 weeks of gestation for malignant hypercalcaemia due to metastatic breast cancer. Two weeks later she gave birth to a healthy male child weighing $3.06 \mathrm{~kg}$. The baby presented transient hypocalcaemia, with full recovery 5 days later. He had normal growth and development. Hypocalcaemia, apart from a direct effect of BP, could also be due to fetal parathyroid suppression by maternal hypercalcaemia. ${ }^{19}$ Furthermore, another report of a woman with metastatic breast cancer in pregnancy treated with intravenous pamidronate at the $28^{\text {th }}$ week of gestation documented no unfavourable impact on the fetus. Fetal calcium level was elevated at birth, subsequently dropped below normal and normalized 9 days after birth. ${ }^{20}$ The third reported case was about a 33-year-old woman with metastatic bone disease due to breast cancer. She was not aware of her pregnancy until the $28^{\text {th }}$ week. Zoledronic acid (4 mg intravenously every 28 days) was administered throughout the second and third trimesters. She gave birth to a normal female at 35 weeks of gestation which was followed up for 12 months and presented normal development. ${ }^{21}$ The most recent report describes a 40-year-old multigravida woman diagnosed with metastatic breast cancer at 24 weeks of gestation and who received chemotherapy with unsatisfactory results. At 28 weeks she was given pamidronate for hypercalcaemia. Pamidronate successfully reduced maternal calcium levels and uterine contractions. The neonate presented normal serum calcium and iPTH levels. 22 


\section{b. BPs and lactation}

One report concerning a 39-year-old woman suffering from sympathetic dystrophy dealt with this issue. Intravenous pamidronate $(30 \mathrm{mg})$ was administered at monthly intervals and breast milk was pumped and collected for 48 hours after the infusion. She resumed breastfeeding between pamidronate administrations. Pamidronate was quantified by high performance liquid chromatography (HPLC) with a limit of quantitation of $0.4 \mu \mathrm{mol} / 1$. Pamidronate levels were below detection limits for all samples of milk tested. The baby remained healthy and presented normal growth. ${ }^{23}$ In addition, in the case of the patient suffering from Gaucher's disease mentioned above, breastfeeding for 1 year was not accompanied by adverse effects to the infant's health and developmental rate. ${ }^{17}$ In the report of 4 cases by Chan et al, also described above, women received intravenous pamidronate for 19-46 months before conception and all of them breastfed their babies for 6 weeks to 18 months. The infants remained healthy and no adverse events occurred. ${ }^{9}$ The only adverse infant outcome reported during breastfeeding was transient hypocalcaemia. Specifically, the mother received BPs before conception and the neonate was breastfed for 3 months. At 2 months, investigation of the child revealed asymptomatic hypocalcaemia $(2.10 \mathrm{mmol} / \mathrm{l}$, normal range $2.25-2.65)$. At 5 months of age, normal serum calcium levels and normal long-bone X-ray were documented. ${ }^{11}$

\section{c. Bone scintigraghy during pregnancy using BPs chemically attached by radionuclides}

Although the main issue concerning bone scintigraghy during pregnancy is fetal radiation exposure, there is also concern regarding fetal skeleton uptake of BPs, which are used as the vehicle of the radionuclides. Existing data show different results when bone scan is performed at different stages of gestation. Two pregnant women underwent bone scintigraghy with technetium-99m methylene diphosphonate at 8 or 18 weeks of gestation. Although placenta exhibited marked uptake, fetal uptake was not demonstrated, while, notably, absorbed dose by fetuses was lower than maternal background levels in both cases. ${ }^{24} \mathrm{In}$ addition, another report presenting a 21 -year-old woman, who also underwent bone scan following the same radionuclide injection at 16 weeks of gestation, suggests that there is no fetal uptake. ${ }^{25}$ However, a report on the cases of 2 pregnant women with malignant tumors showed direct skeletal fetal and placental uptake of radionuclide at 30 and 32 weeks of gestation. None of the children presented adverse effects. ${ }^{26}$ When a scan is required for pregnant women, the lowest dose for adequate results should be used.

\section{DISCUSSION}

Our research identified 78 cases of fetuses whose mothers had been exposed to BPs prior to or during pregnancy, with 69 live births. None of the newborns presented serious adverse events caused by BPs. The abortions that occurred were attributed to maternal concomitant diseases and medications.

Concern about BP use in women of childbearing age is related to the increased calcium requirements during pregnancy and lactation, the pharmacokinetic and pharmacodynamic properties of BPs that result in prolonged retention in the skeleton and suppression of bone turnover as well as animal data pointing to transplacental passage of BP and significant maternal and offspring toxicity, albeit at much higher doses compared to those approved in humans.

Gestation is a period with enhanced metabolic requirements for the pregnant woman and her skeleton, predominantly during the third trimester in which calcium is released from the mother's bones to fulfill the increased fetal demands (which are mainly satisfied by increased maternal intestinal calcium absorption). ${ }^{27}$ In the event that the pregnant woman is treated with BPs shortly before conception or during gestation, suppression of bone turnover may result in fetal hypocalcaemia with detrimental consequences to its bone modeling and other cellular procedures in which calcium plays a dominant role. ${ }^{28}$

BPs' similarity to pyrophosphate accounts for their ability to bind with bone hydroxyapatite. Subsequently, they are taken up by osteoclasts where they exert their antiresorptive action. In a recent study by Nancollas et al, the most popular BPs were ranked according to their binding affinities for hydroxyapatite as follows: zoledronate $>$ alendronate $>$ ibandronate $>$ risedronate $>$ etidronate $>$ clodronate. ${ }^{29}$ Furthermore, the accepted comparative potency of BPs to inhibit osteoclast action is: zoledronate $>$ risedronate $>$ ibandronate $>$ alendronate $>$ pamidronate..$^{30}$ Oral 
BPs have extremely low bioavailability and only about $1 \%$ of the administered oral dose is absorbed by the gastrointestinal tract. Of the absorbed amount, $\sim 50 \%$ is distributed to bones, with the remainder quickly excreted (within hours) by the kidneys $(<0.5 \%$ of the oral dose is excreted through bile). ${ }^{31}$ However, the total elimination of BPs from the body is not accomplished via this initial renal excretion but is a multiphasic and long-term procedure owing to their prolonged binding ("burial") to bones (Figure 1). ${ }^{32,33}$

The above factors explain why BPs have long half-lives in the human body in contrast to their relatively short plasma half-lives. Since the mid-1990's, it has been known that after a single intravenous dose of alendronate in postmenopausal women with osteoporosis, who had never been treated with BPs, the estimated skeletal half-life of the BP exceeds 10 years (based on the data from the first 8 to 18 months' follow-up) ${ }^{34}$ Additionally, the observation that pamidronate can be detected in urine of young patients up to 8 years after discontinuation of oral treatment is suggestive of BPs' long-term retention in the skeleton. ${ }^{35}$ Moreover, a recent study evaluating urinary excretion of alendronate and risedronate in postmenopausal women previously treated with these drugs found that alendronate could be detected up to 19 months after drug cessation: urinary levels were inversely related to time of treatment discontinuation and directly related to uNTx levels. However, 5 months after treatment cessation risedronate was not detected, this underlining the known differences of these two commonly used BPs. ${ }^{36}$ Finally, the fact that biochemical markers of bone turnover do not increase immediately and that the rate of bone loss is slow after cessation of treatment with these drugs, by contrast with hormone replacement therapy in postmenopausal women, is also indicative of BPs' retention in bones. ${ }^{37-41}$

In addition, BPs' small molecular weight possibly allows their passage through placenta and, eventually, some action on the fetus. Apart from animal studies, there are reports in the literature of 2 women who underwent bone scintigraghy with ${ }^{99 \mathrm{~m}} \mathrm{Tc}$-methylene diphosphonate while being in late gestation $(>30$ weeks). ${ }^{26}$ In both cases there was radionuclide uptake from both the placenta and the fetus. Generally, placental permeability increases gradually throughout

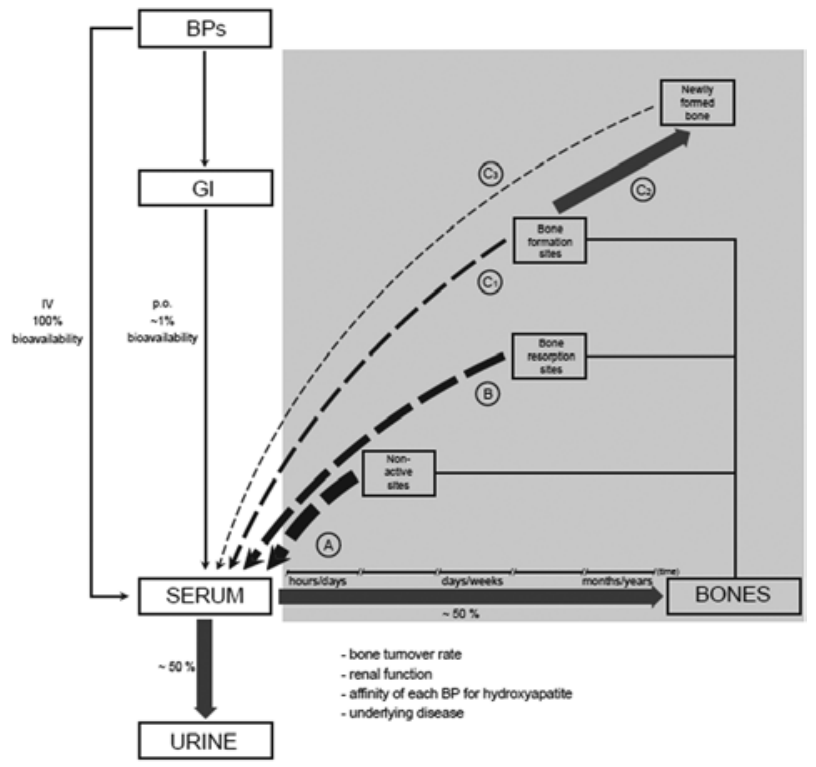

Figure 1. Schematic illustration of BPs' pharmacokinetics. Only $\sim 1 \%$ of the administered oral dose is absorbed by the gastrointestinal tract (GI) and becomes bioavailable; when administered intravenously the bioavailability is $100 \%$. Of the absorbed amount, $\sim 50 \%$ is distributed to bones, while the remainder is excreted quickly (within hours) by the kidneys. ${ }^{31}$ The amount of BPs taken up by the bones depends on the affinity of each BP for hydroxyapatite, the rate of bone turnover (which, in turn, is affected by a variety of factors, such as the underlying disease, age, etc.) and renal function. ${ }^{32}$ The fate of BPs that reach the skeleton depends on the specific binding bone site. A) At non-active (resting) sites ( $\sim 85 \%$ of all surfaces in a certain trabecular segment), BPs are bonded loosely, in small quantities, and return to blood circulation within hours/days, while a very small amount is retained/in bones. ${ }^{33} \mathbf{B}$ ) At sites of bone resorption ( $\sim 2 \%$ of all surfaces in a certain trabecular segment), which have about 8 times greater affinity for BPs than resting sites, the majority of the BPs returns to the blood circulation like non-active sites, but in this case the return lasts longer (days/weeks). ${ }^{33}$ C) Finally, at sites of bone formation ( $12 \%$ of all surfaces of a certain trabecular segment), which have about 4 times higher affinity for BPs than non-active sites, although a significant amount returns to blood within days/weeks (C1), mineralization with the calcium-BPs complexes leads to the incorporation of BPs into the newly generated bone (C2) and their long-term retention in an inert (as far as the bone turnover is concerned) condition, until a new circle of bone resorption liberates them and make them available (C3), either for reuse in bones or for excretion by the kidneys. ${ }^{33}$ This "burial" of BPs is completed within hours/days, but the time these agents remain in this condition cannot be estimated precisely (it may last for years), since it depends on many factors, with bone turnover rate being the most important. 
pregnancy, reaching maximum at around the $32^{\text {nd }}$ week..$^{42}$ Thus, the aforementioned cases should not be extrapolated to BPs' use at earlier stages of gestation. They are however indicative of BPs' passage through placenta. BPs are potentially capable of directly disturbing bone modeling by acting on bone cells or indirectly through hypocalcaemia. Furthermore, recent data have shown an inhibitory effect of BPs on angiogenesis, thus an analogous harmful action is also possible on the fetus. ${ }^{33}$

The above issues highlight the complexity and the difficulty in handling women of reproductive potential needing BP treatment. Judging by the published cases, it seems that BP administration at the approved doses even during the first trimester of pregnancy does not result in significant toxicity. Even in the limited number of cases of malignancy-associated hypercalcaemia, intravenous BP use did not lead to significant infant or neonatal morbidity. Moreover, in cases of OI and PFD, extremely high dose BP treatment did not result in infant harm, apart from transient, reversible or easily treated hypocalcaemia. Thus, it seems that animal data, where the doses used were 10 to 100 times higher than the approved human (alendronate: $10 \mathrm{mg} / \mathrm{kg} /$ day in rats compared to $0.15 \mathrm{mg} / \mathrm{kg} / \mathrm{day}$ based on a patient weight of $70 \mathrm{~kg}$ ), do not apply in humans.

The lack of adequate data and the deficient quality of the published studies have led the US Food \& Drug Administration to place most of the BPs in class C, i.e. animal reproduction studies have shown an adverse effect on the fetus and there are no adequate and well-controlled studies in humans, but potential benefits may warrant use of the drug in pregnant women despite potential risks.

Taking all the above into account, it can be concluded that BP treatment during pregnancy will probably result in fetal exposure. On the other hand, human data do not indicate significant toxicity even in women treated in late gestation, thus termination of pregnancy should most likely not be advocated. Moreover, in women of reproductive potential and with short-term increased fracture risk, such as temporary glucocorticoid treatment, it might be sensible to use BPs with low skeletal retention, such as rise- dronate, balancing it against the relative antifracture efficacy. ${ }^{43}$ However, in cases of high fracture risk, such as OI or PFD, we advocate intravenous BPs administration to attain maximal suppression of high bone turnover in order to protect the maternal skeleton and reduce BPs recycling.

\section{CONCLUSIONS}

The safety of BP use by women of reproductive age has not as yet been fully established. There is evidence that BPs cross the placenta. When the administration of BP concerns women of childbearing age, their long-lasting retention and slow release back to systemic circulation might lead to exposure of the fetus. Animal studies have shown adverse effects both on the fetus and the mother, mostly at doses much higher than those commonly used in humans. Protracted parturition, maternal mortality, embryolethality, severe general underdevelopment and marked skeletal retardation of the fetuses (increased amount of diaphyseal bone trabeculae, decreased diaphyseal length), small fetal weight and abnormal tooth growth have been observed. Conversely, human reports regarding women exposed to BPs before conception or during pregnancy did not demonstrate serious adverse effects either to fetuses or to the mothers. However, there are cases of shortened gestational age, low neonatal birth weight and transient hypocalcaemia. While most authors warn against BPs administration prior to or during gestation, this recommendation is hard to apply in clinical practice. Some suggest that women treated with BPs and who are planning pregnancy should withhold medication for at least 1 year. ${ }^{44}$ Our policy is to withhold medication for at least 6 months, especially in cases of high fracture risk such as OI or PFD. In these patients we advocate intravenous BPs administration to attain maximal suppression of high bone turnover in order to protect maternal skeleton and reduce BPs recycling. Thus, each case should be managed taking into account the risk/benefit balance. Nevertheless, monitoring serum calcium levels during pregnancy and lactation and during the first weeks of the infant's life is imperative for the successful outcome of pregnancy.

No conflict of interest or financial support declared. 


\section{REFERENCES}

1. Top 200 brand-name drugs by units in 2006."www.drugtopics.com/drugtopics/Pharmacy +Facts+And+Figures/ Top-200-brand-name-drugs-by-units-in-2006.

2. Drake MT, Clarke BL, Khosla S, 2008 Bisphosphonates: mechanism of action and role in clinical practice. Mayo Clin Proc 83: 1032-1045.

3. Patlas N, Golomb G, Yaffe P, Pinto T, Breuer E, Ornoy A, 1999 Transplacental effects of bisphosphonates on fetal skeletal ossification and mineralization in rats. Teratology 60: 68-73.

4. Minsker DH, Manson JM, Peter CP, 1993 Effects of the bisphosphonate, alendronate, on parturition in the rat. Toxicol Appl Pharmacol 121: 217-223.

5. Graepel P, Bentley P, Fritz H, Miyamoto M, Slater SR, 1992 Reproduction toxicity studies with pamidronate. Arzneimittelforschung 42: 654-667.

6. Ornoy A, Wajnberg R, Diav-Citrin O, 2006 The outcome of pregnancy following pre-pregnancy or early pregnancy alendronate treatment. Reprod Toxicol 22: 578-579.

7. Levy S, Fayez I, Taguchi N, et al, 2009 Pregnancy outcome following in utero exposure to bisphosphonates. Bone 44: 428-430.

8. Losada I, Sartori L, Di Gianantonio E, Zen M, Clementi M, Doria A, 2010 Bisphosphonates in patients with autoimmune rheumatic diseases: Can they be used in women of childbearing age? Autoimmun Rev 9: 547552.

9. Chan B, Zacharin M, 2006 Maternal and infant outcome after pamidronate treatment of polyostotic fibrous dysplasia and osteogenesis imperfecta before conception: a report of four cases. J Clin Endocrinol Metab 91: 2017-2020.

10. Munns CF, Rauch F, Ward L, Glorieux FH, 2004 Maternal and fetal outcome after long-term pamidronate treatment before conception: a report of two cases. J Bone Miner Res 19: 1742-1745.

11. Hassen-Zrour S, Korbaa W, Bejia I, Saidani Z, Bergaoui N, 2010 Maternal and fetal outcome after long-term bisphosphonate exposure before conception. Osteoporos Int 21: 709-710.

12. Harsch IA, Hubner RH, Hahn EG, Hensen J, 2001 [Osteoporosis and multiple pregnancy--a case report with positive outcome]. Med Klin (Munich) 96: 402-407.

13. Athimulam S, Chan P, Baker M, Nauta M, Yoong W, 2007 The effect of pregnancy on pre-existing osteopenia: a case report. Arch Gynecol Obstet 276: 175-177.

14. Vujasinovic-Stupar N, Pejnovic N, Markovic L, Zlatanovic M, 2011 Pregnancy-associated spinal osteoporosis treated with bisphosphonates: long-term follow-up of maternal and infants outcome. Rheumatol Int, in press.

15. Cabar FR, Nomura RM, Zugaib M, 2007 Maternal and fetal outcome of pamidronate treatment before concep- tion: a case report. Clin Exp Rheumatol 25: 344-345.

16. Rutgers-Verhage AR, deVries TW, Torringa MJ, 2003 No effects of bisphosphonates on the human fetus. Birth Defects Res A Clin Mol Teratol 67: 203-204.

17. Mastaglia SR, Watman NP, Oliveri B, 2010 Intravenous bisphosphonate treatment and pregnancy: its effects on mother and infant bone health. Osteoporos Int 21: 1959-1962.

18. Biswas PN, Wilton LV, Shakir SA, 2003 Pharmacovigilance study of alendronate in England. Osteoporos Int 14: 507-514.

19. Dunlop DJ, Soukop M, McEwan HP, 1990 Antenatal administration of aminopropylidene diphosphonate. Ann Rheum Dis 49: 955.

20. Illidge TM, Hussey M, Godden CW, 1996 Malignant hypercalcaemia in pregnancy and antenatal administration of intravenous pamidronate. Clin Oncol (R Coll Radiol) 8: 257-258.

21. Andreadis C, Charalampidou M, Diamantopoulos N, Chouchos N, Mouratidou D, 2004 Combined chemotherapy and radiotherapy during conception and first two trimesters of gestation in a woman with metastatic breast cancer. Gynecol Oncol 95: 252-255.

22. Culbert EC, Schfirin BS, 2006 Malignant hypercalcemia in pregnancy: effect of pamidronate on uterine contractions. Obstet Gynecol 108: 789-791.

23. Siminoski K, Fitzgerald AA, Flesch G, Gross MS, 2000 Intravenous pamidronate for treatment of reflex sympathetic dystrophy during breast feeding. J Bone Miner Res 15: 2052-2055.

24. Hedrick WR, DiSimone RN, Wolf BH, Langer A, 1998 Absorbed dose to the fetus during bone scintigraphy. Radiology 168: 245-248.

25. Palestro CJ, Malat J, Collica CJ, Richman AH, 1986 Incidental diagnosis of pregnancy on bone and gallium scintigraphy. J Nucl Med 27: 370-372.

26. McKenzie AF, Budd RS, Yang C, Shapiro B, Hicks RJ, 1994 Technetium-99m-methylene diphosphonate uptake in the fetal skeleton at 30 weeks gestation. J Nucl Med 35: 1338-1341.

27. Black AJ, Topping J, Durham B, Farquharson RG, Fraser WD, 2000 A detailed assessment of alterations in bone turnover, calcium homeostasis, and bone density in normal pregnancy. J Bone Miner Res 15: 557-563.

28. Kovacs C, 2006 Skeletal physiology: fetus and neonate. Primer on the metabolic bone diseases and disorders of mineral metabolism, pp; 50-55.

29. Nancollas GH, Tang R, Phipps RJ, et al, 2006 Novel insights into actions of bisphosphonates on bone: differences in interactions with hydroxyapatite. Bone 38: 617-627.

30. Dunford JE, Thompson K, Coxon FP, et al, 2001 Structure-activity relationships for inhibition of farnesyl diphosphate synthase in vitro and inhibition of bone resorption in vivo by nitrogen-containing bisphosphonates. J Pharmacol Exp Ther 296: 235-242. 
31. Lin JH, 1996 Bisphosphonates: a review of their pharmacokinetic properties. Bone 18: 75-85.

32. Cremers SC, Pillai G, Papapoulos SE, 2005 Pharmacokinetics/pharmacodynamics of bisphosphonates: use for optimisation of intermittent therapy for osteoporosis. Clin Pharmacokinet 44: 551-570.

33. Kimmel DB, 2007 Mechanism of action, pharmacokinetic and pharmacodynamic profile, and clinical applications of nitrogen-containing bisphosphonates. J Dent Res 86: 1022-1033.

34. Khan SA, Kanis JA, Vasikaran S, et al, 1997 Elimination and biochemical responses to intravenous alendronate in postmenopausal osteoporosis. J Bone Miner Res 12: 1700-1707.

35. Papapoulos SE, Cremers SC, 2007 Prolonged bisphosphonate release after treatment in children. N Engl J Med 356: 1075-1076.

36. Peris P, Torra M, Olivares V, et al, 2011 Prolonged bisphosphonate release after treatment in women with osteoporosis. Relationship with bone turnover. Bone 49: 706-709.

37. Bagger YZ, Tanko LB, Alexandersen P, Ravn P, Christiansen C, 2003 Alendronate has a residual effect on bone mass in postmenopausal Danish women up to 7 years after treatment withdrawal. Bone 33: 301-307.
38. Landman JO, Hamdy NA, Pauwels EK, Papapoulos SE, 1995 Skeletal metabolism in patients with osteoporosis after discontinuation of long-term treatment with oral pamidronate. J Clin Endocrinol Metab 80: 3465-3468.

39. Bone HG, Hosking D, Devogelaer JP, et al, 2004 Ten years' experience with alendronate for osteoporosis in postmenopausal women. N Engl J Med 350: 1189-1199.

40. Black DM, Schwartz AV, Ensrud KE, et al, 2006 Effects of continuing or stopping alendronate after 5 years of treatment: the Fracture Intervention Trial Long-term Extension (FLEX): a randomized trial. JAMA 296: 2927-2938.

41. Watts NB, Chines A, Olszynski WP, et al, 2008 Fracture risk remains reduced one year after discontinuation of risedronate. Osteoporos Int 19: 365-672.

42. Guyton A 1992. Medical Physiology, Paschalidis eds, Athens; pp, 1205-1207.

43. Reid DM, Devogelaer JP, Saag K, et al, 2009 Zoledronic acid and risedronate in the prevention and treatment of glucocorticoid-induced osteoporosis (HORIZON): a multicentre, double-blind, double-dummy, randomised controlled trial. Lancet 373: 1253-1263.

44. Ott SM, 2004 Effects of bisphosphonates during pregnancy.http://courses.washington.edu/bonephys/oppregnancy.html\#Bis. 2010 November, 26. 